\title{
VAGINAL DELIVERY OF THE TRIPLETS
}

\section{Saha $\mathbf{R}^{1}$, Bastola $\mathbf{N}^{1}$}

\section{ABSTRACT}

The spontaneous conception \& delivery of triplets occurs in 1: 6889 births. Most of the literature recommends caesarean section for triplets for better perinatal outcome of especially second and third baby, however, we describe vaginally delivered triplets with good perinatal outcome.

Key Words: Triplets, Mode of delivery, Neonatal outcome.

\section{INTRODUCTION}

Greek mythology and the bible give early examples of multiple birth. Romulus and Remus were twins raised by the mother Wolf. Easu and Jacob were the twin boys of Rebekah and Isac. Triplet rate is 1:6889 births. Triplets birth are at much greater risk than singleton in terms of poor birth outcomes. More than 9 of 10 triplet birth are born preterm as compared with $<1$ of 10 singleton birta. The average weight of a triplet newborn is about 1.5 $\mathrm{kg}$. The infant death rate for triplets is 12 times $^{1,2}$ higher that for singleton. Based on their frequency of preterm birth, low birth weight, infant death rate, it is appropriate to characterize all triplet pregnancies high risk. ${ }^{3}$ Comprehensive and aggressive prenatal care to assure the best outcomes should be the goal for clinicians caring for these women.

\section{CASE REPORT}

24 years old second gravida at 33 weeks +6days with triplet pregnancy was admitted in active labor on $6^{\text {th }}$ September, 2002 at Kathmandu Medical College Teaching Hospital. She was diagnosed to have triplet pregnancy from 12 weeks of gestation, by an ultrasonography which showed trichorionic and triamniotic placenta. She had her ante-natal checkup in private clinic. There was no family history of multiple pregnancy or history of ovulation induction. Earlier she had pregnancy loss at 6 months of gestation.

1. Kathmandu Medical College Teaching Hospital, Sinamangal, Kathmandu, Nepal.

Address for correspondence : Dr. Rachana Saha, Lecturer

Department of Obstetrics and Gynaecology

Kathmandu Medical College Teaching Hospital, Sinamangal

P. O: 5919, Kathmandu, Nepal 
On admission she was found to be hypertensive with blood pressure of 140/100 mmHg. On obstetric examination the abdomen was hugely distended with multiple parts. The lie of the $1^{\text {st }}$ baby was cephalic, the presentation of the two other babies were difficult to make out. All three fetal heart sound was audible with Doppler. Per vaginal examination showed cervical os dilated up to $5 \mathrm{~cm}$, cervical effacement $60 \%$, membrane present and vertex at +1 station. USG showed all the three babies (in three different sac) to be in cephalic

labeled as one. Immediately thereafter lie and Foetal Heart Rate of other foetures were checked. Per vaginal examination was done which showed cervical os to be fully dilated, cervical effacement $100 \%$ membrane ' + ' and vertex at +2 station. Rupture of membrane was done which showed presence of clear liquor and second alive male baby weighing $1.485 \mathrm{~kg}$ was delivered with apgar score of $6 / 10,8 / 10$. The cord was clamped and cut and the baby was labeled as two. The lie and foetal heart rate was rechecked for the third foetus and per vaginal examination was done which showed fully dilated cervical os with cervical effacement $(100 \%)$, membrane ' + and vertex at +2 station. Artificial rupture of membranes was done which showed clear liquor. Third baby alive female was born after two minutes of the delivery of second baby $1.35 \mathrm{~kg}$ with apgar score $6 / 10,8 / 10$. The cord was clamped and cut and labeled as three. After 15 minutes,

presentation and longitudinal lie. The decision was to allow them for vaginal delivery. Blood sample was taken for cross-matching and vein was kept open with Ringer Lactate. Patient was given Cap. Nifedipin $10 \mathrm{mg}$ orally. Fetal heart rate was monitored every half hourly with Doppler. After 4 hours she was examined internally to monitor the progress of labor. The cervix was dilated up to $8 \mathrm{~cm}$, cervical effacement was $100 \%$, and vertex was at +2 station. Artificial rupture of membrane was done which showed clear liquor. At crowning, episiotomy was given. A live male baby weighing $1.43 \mathrm{~kg}$ with apgar score of $6 / 10,8 / 10$ was born. The cord was clamped and cut immediately and the placenta was delivered and inj. Syntocinon 5 IU bolus and 10 IU in drip was kept. Total blood loss was $140 \mathrm{ml}$. Post delivery blood pressure of mother was $140 / 100$. Babies were transferred to neo-natal intensive care.

The maturity of all babies was 32 weeks. All the babies had good sucking but later they developed jaundice for which they received phototherapy and antibiotics. They were discharged after 27 day with weight of the baby first being $1.75 \mathrm{~kg}$, second baby $1.75 \mathrm{~kg}$, and third baby $1.95 \mathrm{~kg}$. Post partum stay of the mother was uneventful and she was discharged on Cap. Amodipine $5 \mathrm{mg}$ daily. 
DISCUSSION

Labour and delivery of triplets entails increased risk as well. Fetal heart rate monitoring during labor is challenging with vaginal delivery, the first infant is usually born spontaneously or with little manipulation. However, subsequent infants are delivered according to the presenting part, which often require complicated obstetrical maneovres such as total breech extraction with or without podalic version and many even necessitate caesarean delivery. Associated with appositions is increased incidence of cord prolapses and fetal collision. For all these reasons, many clinicians believe that delivery of triplets is best accomplished by caesarean section. ${ }^{3}$

Caesarean section was the mode of delivery in $84 \%$ of cases of triplets in the series of 86 triplets delivered in Sweden in 1985 and 1989. ${ }^{4}$ Similarly more liberal approach towards abdominal delivery of pregnancies of higher fetal number is advocated in the retrospective review of triplets delivered at the Boston perinatal center from 1976 to 1986 because of the increased morbidity from $1^{\text {st }}$ to $2^{\text {nd }}$ and $3^{\text {rd }}$ twin delivered vaginally ${ }^{5}$

Lipitz et al $^{6}$ in their study of 78 triplets from 1975 to 1978 and Growther CA and, Hamilton RA ${ }^{7}$ in their 105 cases of triplets from 1975-1984 also have recommended caesarean section.

Vaginal delivery is reserved for those circumstances in which survival is not expected because the fetus are markedly immature, or maternal complication make caesarean delivery hazardous to the mother. But then there are other clinicians who believe that vaginal delivery is safe under certain circumstances. For example - Alaniia and colleagues 1998 evaluated a protocol for vaginal delivery. A total of 23 sets of triplets were analyzed and a third were delivered vaginally. Neonatal outcomes were similar in both groups. ${ }^{3}$ Similar observation was done by Ron eir ${ }^{8}$ in their management of 19 triplets during the period of 1970-1978.

In this case we allowed vaginal delivery because the triplets were triamniotic, trichorionic, all three babies were in cephalic presentation and they were at 33 week of gestation and in active labour. Vaginal delivery fortunately did not bring any untoward complication in mother and immediate complication to the baby.

\section{BIBLIOGRAPHY}

1. $K$ eith $L G, 0$ leszcute J I. T riplets birth in the $U$ nited $S$ tates. A $n$ epidemic og high risk pregnancy. J. $R$ eprod M ed 2002 A pril 47(4): 259-265.

2. $M$ artin $\int A, M$ ac $D$ orman $M F, M$ athew $T$ I. T riplets birth trends and outcomes 1971-74. V ital health stat $21^{\star} 1997$ J an: (55); 1:20.

3. $M$ ultifoetal pregnancy in $C$ unningam $F G, F G$ $\mathrm{N}$ orman, $\mathrm{H}$ eveno $\mathrm{K} J$ : $G$ ilstrap $L \mathrm{C}, \mathrm{H}$ anth $\mathrm{C} J$, $W$ enstrom $D K$ edited $W$ illiams obstetrics (21 $1^{\text {st }}$ eation), U SA ,M C-G R A W H I L L 2001.

4. $\mathrm{R}$ adsted $\mathrm{A}$. B irth of tripl ets quadrupl ets in $\mathrm{S}$ weden $N$ ord M ed 1991; 106 (11) 297-298.

5. F eingold $M, C$ etrul $O C, P$ eters $M$, et al. mode of delivery in multiple birth in high order. $A$ cta $G$ enet $M$ ed $G$ emellol (R oma) 1988; 37(1); 105-9.

6. L ipitz $S, R$ eichman $B, P$ aret $G$, et al. T heimproving outcome of triplet pregnancies. A M.J 0 bstet G ynecol 1989 N OV., 161(5): 1979-84.

7. G rowther $C A, H$ amilton $R A$. T riplet pregnancy: a ten-year review of 105 cases at $\mathrm{H}$ arare maturity hospital $Z$ imbabwe. A cta $G$ enet $M$ ed $G$ emellol (R oma) 1989; 38(3-4): 271-8.

8. $R$ on- $E \mid R, C$ aspi $E, S$ chreyer $P$ et al. $T$ riplet and quadruplet preganacies and management. 0 bstet $G$ ynecol 1981 A pril 57(4):458-63.

$$
\text { 为为为 }
$$

